\title{
Uso de sustancias y percepción de riesgo: Estudio comparativo entre jóvenes de Bogotá y Barcelona
}

\author{
Ángela María Trujillo*; María Forns I Santacana**; Augusto Pérez Gómez*** \\ *Universidad de Barcelona, Doctoranda, Becaria FI de la Generalitat de Catalunya. \\ **Universidad de Barcelona. Catedrática. \\ ***Corporación Nuevos Rumbos, Bogotá. Director. \\ Enviar correspondencia a: \\ Ángela M. Trujillo. Passeig de la Vall d’Hebrón, 171 6ta planta. 08035 Barcelona, España. atrujilloc@ub.edu.
}

Recibido: Julio de 2006. Aceptado: Febrero de 2007

\section{RESUMEN}

Este artículo intenta determinar las diferencias y similitudes existentes entre jóvenes de Barcelona y jóvenes de Bogotá, en cuanto a sus hábitos de consumo de sustancias psicoactivas, la percepción que tienen sobre actividades que involucran el consumo y cómo la percepción de riesgo influye en estos hábitos. La muestra la componen 865 jóvenes de ambas ciudades escolarizados, entre los 15 y los 18 años. Se utilizaron las variables de riesgo estudiadas por Benthin, Slovic y Severson (1993) para evaluar la percepción de riesgo y los hábitos de consumo se evaluaron mediante las respuestas de los jóvenes a preguntas sobre la edad en la que consumieron por primera vez alguna de las sustancias, frecuencia de consumo en la última semana y la intención de consumo en el siguiente año. Se encontró que la edad de inicio del alcohol, marihuana y la primera vez que se embriagan, es significativamente diferente según la ciudad en la que se vive. Los jóvenes de Barcelona tienen una propensión y un consumo real mayor que los jóvenes de Bogotá, en cuanto a todas las sustancias evaluadas. Percibir placer o beneficios predice un incremento en la intención y la frecuencia de consumo de la mayoría de las sustancias a excepción de los jóvenes mayores (17 a 18 años) de Bogotá. El miedo a las consecuencias, o el percibir riesgo a enfermarse o herirse y la facilidad para acceder a las sustancias no parecen tener una influencia clara en los diferentes hábitos de consumo.

Palabras clave: Uso de sustancias, percepción de riesgo, estudio comparativo, prevención, adolescentes

\section{ABSTRACT}

The purpose of this article is to determine the differences and similarities between adolescents from Barcelona and Bogotá in their psychoactive substance use habits, their perception of the activities that involve this use and how their risk perception can influence these habits. The sample consists of 865 school students from both cities, with ages between 15 and 18 years. Risk perception was assessed using the risk variables studied by Benthin, Slovic and Severson (1993). Substance use habits were assessed on the basis of the answers given by the youngsters to questions on the age when they first used any substance, frequency of use in the last week and use intentions in the next year. The findings suggest that the age they begin to use alcohol and marijuana and the first time they get drunk differ significantly between the adolescents in the two cities. The Barcelona youngsters have a higher tendency and actual use in respect of all the substances studied than the Bogotá youngsters. Perceived pleasure or benefits predict an increase in both the intention and frequency of use for almost every substance in this study for all, with the exception of the older adolescents (17 to 18 year-olds) from Bogotá. Fear of the consequences or the perception of a risk of illness or injury and conditions favouring use do not seem to have an obvious influence on the various use habits.

Key words: Substance abuse, risk perception, comparative study, prevention, adolescence.

\section{INTRODUCCIÓN}

$\mathbf{L}$ os datos del Observatorio Español sobre Drogas, en su Informe de 2004 (PNSD, 2004) ponen de relieve que en España el patrón de consumo dominante entre la población juvenil es el esporádico, asociado a contextos lúdicos. En Colombia, el estudio llevado a cabo por Rumbos en 2001, referido a jóvenes entre los 10 y los 24 años, mostró que es mayor el porcentaje de jóvenes que han probado una droga alguna vez, que los que la consumen actualmente (la relación es aproximadamente de 2:1). 
En España se ha encontrado que el fumar tabaco se establece, normalmente, en la adolescencia siendo las mujeres quienes fuman más (PNSD, 2004). Este estudio muestra que la edad a la que los jóvenes entre 14 y 18 años fuman su primer cigarrillo de tabaco es 13,2 y la proporción de fumadores aumenta con la edad. En Colombia el consumo de tabaco comienza a los 13,7 años de edad en jóvenes entre los 10 y los 21 años y son los hombres quienes más lo consumen (Rumbos, 2001)

En cuanto al alcohol, en la sociedad española el consumo de alcohol es muy elevado en adolescentes entre 15 y 17 años, tanto en cantidad como en frecuencia, al igual que la frecuencia de episodios de intoxicación (Ballester, Gil y Girardo, 2000; PNSD, 2004; Sánchez, 2000). Amengual, Calafat y Palmer (1993), encontraron que en los jóvenes entre 14 y 18 años hay un $86 \%$ que han bebido alcohol al menos una vez y que el consumo medio de estos jóvenes va aumentando con la edad. Adicionalmente observaron que existe un aumento de su consumo en fines de semana y que el $25 \%$ llegan a la embriaguez. Según el PNSD (2004), la edad de inicio en el alcohol para jóvenes entre los 14 y los 18 años es 13,7. Por su lado, en Colombia el estudio de Rumbos (2001) muestra que el 83,8\% de los jóvenes han bebido alcohol alguna vez en la vida y la edad de inicio está alrededor de los 13 años.

Con respecto al cannabis, un estudio llevado a cabo por Parker, Aldridge y Measham (1998), puso de relieve que en jóvenes británicos de 14 años el consumo de cannabis está alrededor del 30\%. Sin embargo, cuando se estudia el consumo en jóvenes de 18 años, el consumo aumenta a un 60\%. Los autores mostraron que ocurre lo mismo con otro tipo de drogas, como las anfetaminas, que aumentan en un $23 \%$, la cocaína que pasa de un $1,4 \%$ a un $6 \%$, la heroína de un 0,4\% a un 6\% y el éxtasis de un 5,8\% a un $20 \%$. Según el PNSD (2004), la edad en que los jóvenes entre los 14 y los 18 años inician el consumo de marihuana es 14,7 años y el 42,7\% la han consumido alguna vez en la vida. Por su lado, la edad de inicio en sustancias como la cocaína, heroína y el éxtasis está cerca a los 15 años. En Colombia, según Rumbos (2001), el uso de marihuana comienza a los 14,8 años y cerca del $9 \%$ la han consumido alguna vez en la vida. En cuanto a la cocaína, la heroína y el éxtasis, la edad de inicio en Colombia está por encima de los 14,5 años.

Los estudios epidemiológicos llevados a cabo en varios países, muestran que aunque el alcohol suele ser la sustancia más común entre los adolescentes, la prevalencia del uso de marihuana es mayor a la de cualquier otra sustancia ilegal (Makkai y McAllister, 1998; Rumbos, 2001; Johnston, O'Malley, Bachean y Schulenberg, 2004). Los datos acumulados publicados en los últimos años por las Naciones Unidas muestran exactamente la misma tendencia (UNODC, 2006).

\section{Percepción de riesgo en el uso de sustancias}

El consumo de sustancias psicoactivas, entre otros comportamientos como el sexual o los desórdenes de alimentación, delincuencia, deporte, homicidios y suicidios, ponen en peligro la salud y el bienestar de los adolescentes (Ballester et al. 2000). En este estudio nos centramos en el uso de sustancias como el alcohol, el tabaco, la marihuana, la cocaína, la heroína y las pastillas. Todas estas sustancias psicoactivas tienen efectos sobre la salud del adolescente de forma directa (sobredosis, enfermedades cardiovasculares) e indirecta (accidentes de tráfico, deterioro familiar). Los jóvenes, aún siendo conscientes de estas posibles consecuencias negativas, tienden a experimentar con este tipo de actividades de alto riesgo. Parker et al. (1998) argumentan que el consumo no se halla sólo ligado a una respuesta pasiva frente a la oportunidad y/o incentivo de uso, sino que las decisiones sobre el uso de drogas están relacionadas con las apreciaciones de beneficio y de riesgo que el consumo supone. En efecto, se ha demostrado que hay adolescentes que rechazan ciertas drogas, a pesar de las oportunidades que tienen para utilizarlas (Fountain, Griffiths, Farrell, Gossop, y Strang, 1999). Por ello es importante entender qué hace que un joven se involucre en estas actividades, mientras que otro no lo haga.

Diversas investigaciones avalan el rol que la percepción de riesgo toma para el consumo. Así, Benthin, Slovic y Severson, (1993), proponen que el consumo se presenta por la influencia de las percepciones y actitudes sobre los riesgos asociados a una actividad en particular. Calafat, Fernández, Juan, Bellis, Bohrn et al., (2001) encuentran que la percepción de riesgo y la predisposición al riesgo, son determinantes importantes para que un joven tenga un comportamiento de alto riesgo. Benthin et al. (1993) examinaron la relación entre percepción de riesgo y la participación en actividades de alto riesgo, encontrando que los adolescentes que llevan a cabo este tipo de actividades, suelen reportar una percepción de riesgo menor. Por su parte, Mayock (2002) identificó que el miedo a las consecuencias negativas de las actividades de riesgo es dominante para abstenerse de ejecutarlas. Al comparar consumidores con no consumidores se evidenció que los no consumidores suelen sobrevalorar las posibles consecuencias negativas que surgen del uso de sustancias, en comparación a cualquier beneficio. En contrapartida, en el estudio de IREFREA (Calafat, Stocco, Mendes, Simon, Van de Wijngaart et al. 1998), sobre el uso de éxtasis se halló que los consumidores de esta sustancia valoraron positivamente sus efectos y subvaloraron los peligros que se asocian a su uso. 
La medición de la percepción de riesgo ha sido abordada por Hampson, Severson, Burns, Slovic y Fisher (2001). Estos autores han desarrollado una medida de la percepción de riesgo para adolescentes a partir de la escala de percepción de riesgos tecnológicos para adultos (Slovic, 1987). Mediante esta medida, han evaluado las percepciones de los adolescentes sobre diferentes facetas de los riesgos y los beneficios asociados a una gran variedad de actividades de alto riesgo, tanto lícitas como ilícitas, típicas de la edad que se está evaluando (Benthin, et al., 1993; Severson, Slovic, Hampson, y Schrader, 1990). En el presente estudio se ha utilizado esta aproximación para ealuar la percepción de riesgo de actividades relacionadas con el uso de sustancias psicoactivas y poder así determinar qué efecto tiene sobre los hábitos de consumo de los adolescentes.

Los objetivos de este estudio son: a) explorar las diferencias en cuanto a la edad de inicio y los hábitos de consumo de jóvenes entre los 15 y los 20 años de Bogotá (Colombia) y Barcelona (España) y, b) establecer la influencia de la percepción de 9 variables de riesgo en los hábitos de consumo controlando el contexto cultural.

\section{MÉTODO}

\section{Procedimiento}

En ambas ciudades, una vez identificados los colegios públicos y privados de diferentes zonas de las ciudades que cubrieran en lo posible las diferentes clases sociales, se les contactó para pedir la colaboración del colegio en la investigación. En Barcelona participaron diez instituciones y en Bogotá participaron seis. Se recopilaron datos de jóvenes de primero y segundo de bachillerato y de formación profesional de grado medio de Barcelona. En Bogotá se recopilaron datos de noveno, décimo y once de bachillerato, que son equivalentes en edad a los de Barcelona. Se obtuvo, entonces, el consentimiento por parte de la dirección y de los padres de los jóvenes. Se controló la edad de los adolescentes, seleccionando aquellos comprendidos entre 15 y 18 años, ambos inclusive. En el momento de la recolección de los datos se explicó a los alumnos la importancia de su colaboración y se les invitó a que participaran voluntariamente en el proyecto, al mismo tiempo que se les garantizó el anonimato de sus respuestas. Ninguno de los alumnos se negó a colaborar. La aplicación del cuestionario se realizó en las aulas, durante las horas de tutorías o clases y tomó aproximadamente 45 minutos. La aplicación fue realizada por uno de los autores de la investigación. Se eliminaron 6 cuestionarios por presuntas irregularidades en las respuestas.

\section{Muestra}

La muestra se compone de 865 jóvenes de las ciudades de Bogotá $(n=469)$ y Barcelona $(n=396)$, escolarizados, entre los 15 y los 18 años de edad. En la tabla 1 puede apreciarse la distribución de la muestra según variables demográficas.

Tabla 1. Descripción de la muestra

\begin{tabular}{|c|c|c|}
\hline Datos demográficos & Muestra & Porcentaje \\
\hline $\begin{array}{l}\text { Edad media (ấos) } \\
\text { Barcelona } \\
\text { Bosond́ }\end{array}$ & $\begin{array}{l}16,37(\text { S.D. }=0,94) \\
16,8 \quad(\text { S.D. }=0,78) \\
16 \quad(\text { S.D. }=0,90)\end{array}$ & \\
\hline \multicolumn{3}{|l|}{ Grupos de edad } \\
\hline $\begin{array}{l}15 \text { a } 16 \text { años } \\
17 \text { a } 18 \text { años }\end{array}$ & $\begin{array}{l}499 \\
366\end{array}$ & $\begin{array}{l}58 \% \\
42 \%\end{array}$ \\
\hline \multicolumn{3}{|l|}{ Genero } \\
\hline Hombres Barcelana & 186 & $22 \%$ \\
\hline Hombres Bogotá & 248 & $29 \%$ \\
\hline Mrijeres Barcelona & 208 & $24 \%$ \\
\hline Murjeres Bogotá & 218 & $25 \%$ \\
\hline \multicolumn{3}{|l|}{ Nivel Sociocultural } \\
\hline \multicolumn{3}{|l|}{ Barcelana } \\
\hline Alto & 200 & $51,3 \%$ \\
\hline Media & 105 & $26,9 \%$ \\
\hline Bajo & 85 & $21,8 \%$ \\
\hline \multicolumn{3}{|l|}{ Bogotá } \\
\hline Alto & 176 & $38,3 \%$ \\
\hline Media & 114 & $24,8 \%$ \\
\hline Bajo & 166 & $36,8 \%$ \\
\hline
\end{tabular}

\section{Instrumentos de medida}

Hábitos de consumo: Los hábitos de consumo analizados se refieren al alcohol, el tabaco, la marihuana, la cocaína, la heroína y las pastillas. Se ha recogido información acerca de: a) edad de inicio en una escala Likert de 6 grados que va de 11 años o menos a 16 años o más $y$, adicionalmente se pregunta sobre la edad en que el joven se embriagó por primera vez; b) intenciones de consumo durante el próximo año en una escala Likert de 3 grados, donde 1 es improbable y 3 es muy probable y c) consumo actual en una escala Likert de 5 puntos que va de 0 veces a 11 veces o más. Estas preguntas fueron extraídas y agrupadas por el "European Monitoring Center for Drugs and Drug Addictions" a partir de los estudios de Jessor, Van den Bos, Banderín, Costa y Turbin (1995) y Morgan y Grube (1991).

Percepción de Riesgo: Para evaluar la percepción de riesgo se emplearon algunas de las escalas de Benthin et al. (1993). Con estas escalas se analizaron 4 actividades potencialmente de riesgo a) beber alcohol los fines de semana y en reuniones sociales; b) fumar tabaco mínimo una vez al día; c) fumar marihuana con regularidad -1 o más veces por semana y d) probar drogas como la cocaína, la heroína o pasti- 
llas. Para cada una de estas actividades se evaluaron las 9 variables asociadas a la percepción de la situación (conocimiento del riesgo, miedo, riesgo personal, riesgo a terceros, beneficios, presión, admiración, evitación y facilidad). Cada una de estas variables debía ser puntuada en una escala Likert de 1 a 7, según la percepción de riesgo. Cada actividad era ponderada en las nueve escalas, antes de pasar a la otra actividad (ver tabla 2)

Tabla 2. Variables analizadas para cada una de las actividades de riesgo..

\section{Actividades de riesgo \\ Bebida de alcohol \\ Fumar cigarrillos \\ Fumar Marihuana \\ Tomar drogas fuertes}

\section{Conocimiento de los adolescentes sobre los riesgos}

¿Hasta dónde los riesgos asociados a esta actividad son conocidos por las personas de tu edad? (1= No se conoce mucho el riesgo; 7 = Se conoce muy bien el riesgo)

\section{Miedo a los riesgos}

¿Hasta qué punto los riesgos (daños) potenciales asociados con esta actividad producen miedo a personas de tu edad? $(1$ = No producen ningún miedo; 7 = Muy Miedosos.

\section{Riesgo Personal}

Si hicieras esta actividad ¿hasta qué punto crees que estarías personalmente en riesgo de ser herido o enfermarte? (1 = En mucho riesgo; 7 = En ningún riesgo)

\section{Riesgo a Terceros}

Si alguno de tu edad hiciera esta actividad, ¿hasta qué punto crees que él o ella estaría en riesgo de hacerse daño o enfermarse? (1= En mucho riesgo; 7= En ningún riesgo)

\section{Placer o Beneficios}

Si hicieras esta actividad, ¿hasta qué punto te dará placer u otros beneficios? $(1=$ Muy pocos; $7=$ Muchos)

\section{Presión de Iguales}

¿Hasta qué punto sientes presión de tus amigos para que hagas esta actividad? $(1$ = Ninguna presión; 7 = Mucha presión)

\section{Admiración}

¿Hasta dónde las personas que hacen esta actividad son admiradas por sus amigos? (1 = Para nada admiradas; $7=$ Muy admiradas)

\section{Evitación}

¿Hasta dónde una persona de tu edad evita hacer esto? $(1$ = Lo evita por completo; 7 = No lo evita en absoluto)

\section{Facilidad para hacerlo}

Si quisieras participar en esta actividad, ¿con qué facilidad podrías hacerlo? $(1=$ Para nada fácil; $7=$ Extremadamente Fácil)

\section{RESULTADOS}

Como primera medida se analizaron las diferencias de medias entre las dos ciudades en cuanto a la edad en que iniciaron el consumo de las diferentes sustancias. Adicionalmente se hizo una comparación porcentual entre las dos ciudades para determinar los hábitos de consumo de intención y frecuencia. Por último se llevaron a cabo una serie de regresiones para determinar la relación entre los hábitos de consumo y la percepción de riesgo. Para estas regresiones se emparejaron las variables de la actividad "beber alcohol los fines de semana y en reuniones sociales" con los hábitos de consumo relativos a esta sustancia. Se hizo lo mismo para cada una de las sustancias (tabaco, marihuana y cocaína, heroína o pastillas). La variable sexo se codificó con un valor de 0 para hombres y 1 para mujeres. Para la variable de edad de ini- 
cio, se asumieron los valores de 11 años, cuando el joven marcaba 11 años o menos y 16 cuando marcaba 16 años o más. Para determinar la influencia de la edad de los jóvenes a lo largo del estudio, se repetían los análisis dividiendo la muestra de cada ciudad en dos grupos de edad: jóvenes entre los 15 y 16 años y jóvenes entre los 17 y 18 años, dado que cualquier resultado en conjunto podría estar contaminado por las edades de los jóvenes en las dos ciudades.

\section{Hábitos de Consumo}

Al comparar la edad de inicio de consumo de las diferentes sustancias en ambas ciudades, se ha constatado que la edad de inicio de consumo de alcohol es significativamente menor en Bogotá $(M=12,56$; D.T. $=1,4)$ que en Barcelona ( $M=13,59 ;$ D.T. $=1,56$; $t=9,76 ; p<0,001)$. Adicionalmente, los jóvenes de Bogotá suelen embriagarse por primera vez a una edad más temprana $(M=13,78 ; D . T .=1,5)$ que los de Barcelona $(M=14,61$; D.T. $=1,23 ; t=7,55 ; p<0,001)$. Estas diferencias significativas se mantuvieron cuando se hizo el análisis comparando las dos ciudades por grupo de edad (jóvenes menores - entre los 15 y los 16 años - y jóvenes mayores - entre los 17 y los 18 años). El consumo de marihuana presenta diferencias significativas según edad; los jóvenes entre los
17 y 18 años de Barcelona dicen haberse iniciado en el consumo antes $(M=14,31 ; D . T .=1,37)$ que los de Bogotá $(M=15,23 ;$ D.T. $=1,12 ; t=-3,48 ; p<0,001)$. En el grupo de jóvenes de 15 a 16 años no se encontraron diferencias significativas en cuanto a la edad de inicio de la marihuana la cual se encuentra alrededor de los 14,5. En ambas ciudades la edad aproximada en la que fuman el primer cigarrillo de tabaco es alrededor de los 13 años y la edad en al que prueban por primera vez drogas fuertes como la cocaína, la heroína o las pastillas es a los 15 años y sin que se hayan constado diferencias significativas según los grupos de edad.

La figura 1 muestra la distribución porcentual de los jóvenes según ciudad y grupo de edad, para las variables "intención de consumo en el próximo año" $y$ "frecuencia de consumo durante la semana anterior". En cuanto a la intención de consumo de alguna de las sustancias se puede observar que un alto porcentaje de adolescentes de ambas ciudades afirma que el alcohol es la sustancia con mayor probabilidad de ser consumida en un futuro cercano(60\% y $80 \%$ en Bogota y $71 \%$ y $78 \%$ en Barcelona, según grupo de edad). En cuanto al tabaco, en ambas ciudades la mitad del grupo de adolescentes mayores, dice que existe una alta probabilidad de fumar, y algo más de la mitad de los adolescentes menores afirman que

Intención de consumir en un futuro cercano
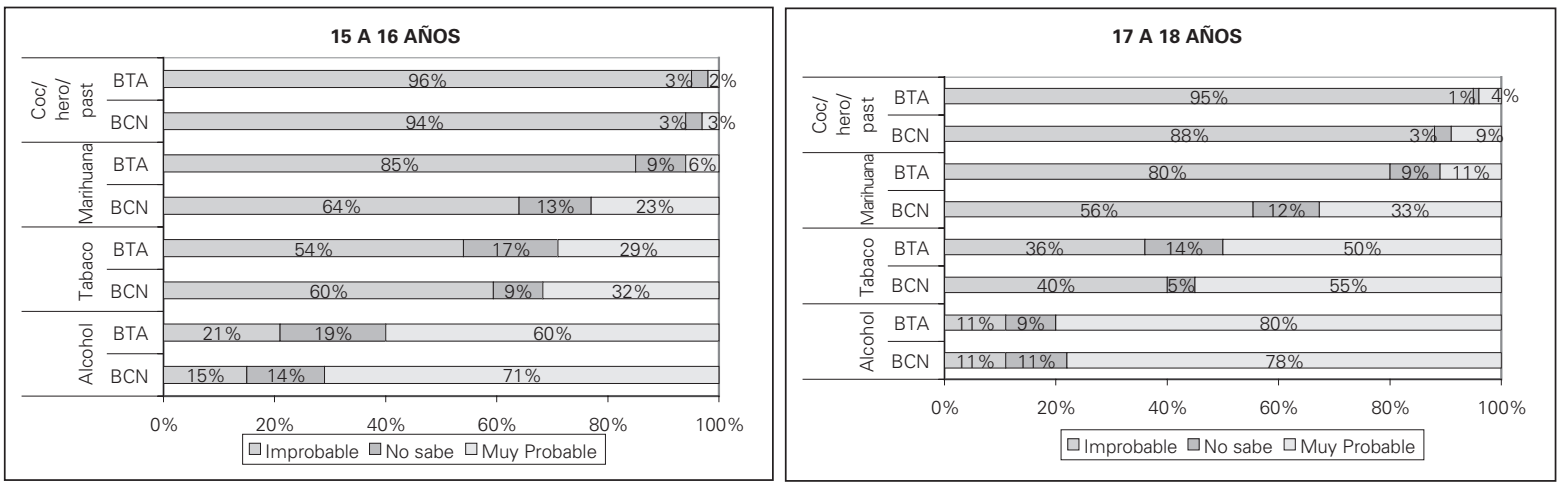

Frecuencia de consumo en la última semana
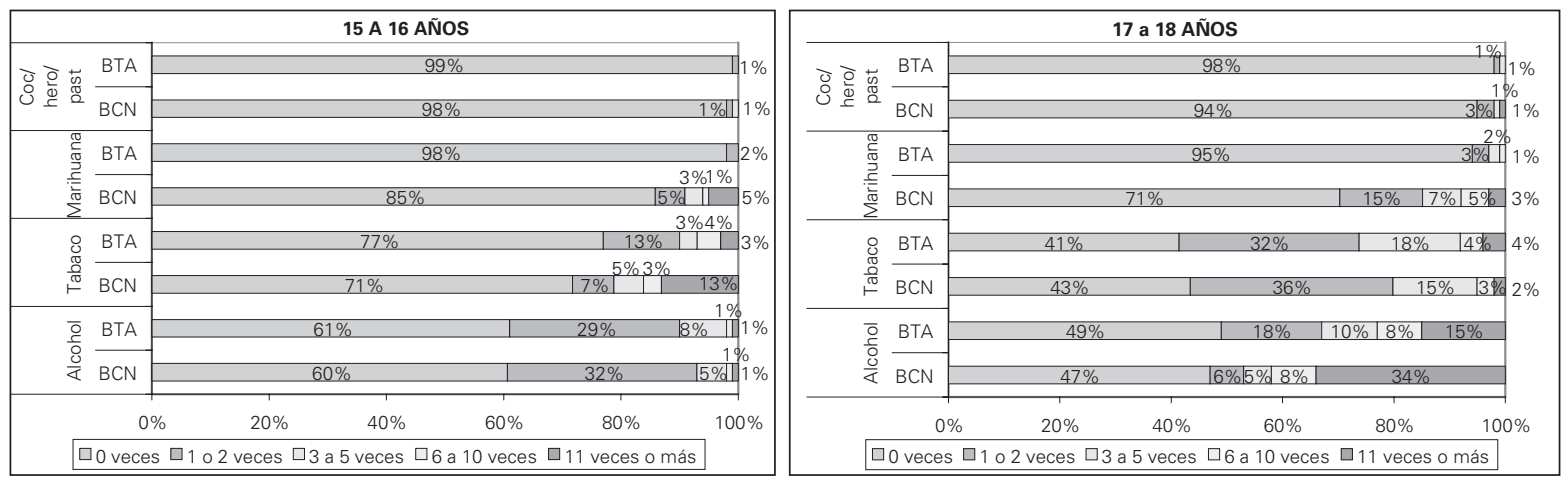

Figura 1. Intención de consumir alguna de las sustancias en un futuro cercano y frecuencia de consumo en la semana anterior a la encuesta para Barcelona y Bogotá, por grupos de edades 
es improbable que fumen en un futuro cercano. En relación a la marihuana es importante resaltar que los adolescentes de Barcelona informan sobre una probabilidad de consumo que alcanza el $23 \%$ entre los más jóvenes y el $33 \%$ entre los mayores, significativamente mayor que las tasas alcanzadas por los adolescentes de Bogotá. Finalmente, en relación al consumo de drogas fuertes, el grupo de adolescentes menores ven una baja posibilidad de consumir drogas fuertes (menos del 3\%) a lo largo del próximo año y es relevante que este porcentaje alcance el $9 \%$ en los adolescentes mayores de la ciudad de Barcelona.

Al analizar la frecuencia de consumo se aprecia que entre los adolescentes más jóvenes para ambas ciudades, aproximadamente el $60 \%$ no ha consumido alcohol y aproximadamente el $75 \%$ no ha consumido tabaco; este porcentaje de no-consumo desciende al $50 \%$ e para el alcohol y al $42 \%$ en el tabaco aproximadamente, en los adolescentes mayores. El consumo de alcohol en una frecuencia superior a tres veces por semana alcanza el $8 \%$ y el $10 \%$ en adolescentes jóvenes de Barcelona y Bogotá respectivamente y el $47 \%$ y el $33 \%$, en adolescentes mayores de BarceIona y Bogotá respectivamente. Se aprecia que en adolescentes mayores de Barcelona, el consumo de alcohol se caracteriza por su uso más regular, con una frecuencia de 34\% de adolescentes que informa haberlo consumido 11 o más veces a lo largo de la semana anterior.

La marihuana por su parte, ha sido consumida durante la semana en mayor proporción por los jóvenes de Barcelona (15\% de los jóvenes entre los 15 y los 16 años y $29 \%$ de los jóvenes entre los 17 y los 18 años), de los cuales más del 6\% lo hicieron seis veces o más. Por su lado, el $2 \%$ de los adolescentes menores de Bogotá afirman haber consumido marihuana y en una frecuencia de 1 ó 2 veces en la semana. En cuanto a los mayores, el 5\% la consumieron pero ninguno afirma haberlo hecho más de 10 veces en la semana. Al observar los porcentajes obtenidos en ambos grupos de edad en las dos ciudades en cuanto al no consumo de las diferentes sustancias, resalta el hecho de que todas las sustancias, durante la última semana, han sido consumidas por un porcentaje un poco mayor de jóvenes en Barcelona que en Bogotá, exceptuando el consumo de tabaco en los jóvenes mayores.

\section{Escalas de percepción de riesgo:}

La tabla 3 presenta las medias y las desviaciones tipo de cada variable de riesgo, para el total de las cuatro actividades relacionadas con el cosnumo.

Existen diferencias significativas entre las dos ciudades en cuanto a la percepción de las características de riesgo de las cuatro actividades, tomadas conjuntamente, excepto en la escala de conocimiento y en la de admiración. Los adolescentes de Barcelona dan valores más elevados en percepción del riesgo personal, riesgo a terceros, obtención de beneficios, evitación y facilidad de consumo. Los adolescentes de Bogota dan valores más elevados en las escalas de miedo y presión. Esto sugiere que los jóvenes de Bogotá en comparación con los de Barcelona, perciben más miedo y sienten más presión por parte de sus iguales para llevar a cabo cualquiera de las cuatro actividades. Los jóvenes de Barcelona sienten en menor medida el riesgo de herirse o enfermarse

Tabla 3. Medias, y desviaciones tipo de cada variable de riesgo, para el total de las cuatro actividades de consumo y diferencias de medias según ciudad (Escalas de 1 a 7).

\begin{tabular}{lccccc}
\hline & \multicolumn{2}{c}{ BARCELONA } & \multicolumn{2}{c}{ BOGOTA } & \multicolumn{2}{c}{ Contraste } \\
\cline { 5 - 6 } Escala & Media & Desv.Típ. & Media & Desv.Típ. & $t$ \\
\hline Conocimiento & 4,93 & 1,35 & 4,99 & 1,57 & $-6,24$ \\
Miedo & 3,18 & 1,09 & 4,31 & 1,34 & $-13,59^{* * *}$ \\
Riesgo Personal & 3,25 & 1,25 & 2,76 & 1,37 & $5,36^{* * *}$ \\
Riesgo Terceros & 3,19 & 1,17 & 2,75 & 1,35 & $5,03^{* * *}$ \\
Beneficios & 2,94 & 1,35 & 2,43 & 1,25 & $5,73^{* * *}$ \\
Presión & 1,56 & 0,95 & 1,81 & 1,12 & $-3,57^{* * *}$ \\
Admiración & 2,33 & 1,30 & 2,15 & 1,26 & 2,17 \\
Evitación & 4,20 & 1,10 & 3,46 & 1,24 & $9,31^{* * *}$ \\
Facilidad & 4,99 & 1,58 & 3,16 & 1,74 & $16,17 * * *$ \\
\hline
\end{tabular}

$* * * p<0,001$

Nota: En algunos casos los puntajes altos se asocian con percepciones bajas de la característica (Ej. Riesgo Personal y terceros). La calificación de cada ítem se da en la tabla 2. 
cuando llevan a cabo estas cuatro actividades de consumo. Cuando se hace esta comparación teniendo en cuenta los dos grupos de edad, se mantienen las diferencias significativas en las escalas. Sin embargo, para los jóvenes entre los 17 y los 18 años, la variable presión ya no difiere entre las dos ciudades. Es decir que esta variación que se encuentra a nivel general es dada principalmente porque los jóvenes Bogotanos entre los 15 y los 16 años sienten mayor presión por parte de sus iguales.

Influencia de la percepción de riesgo en los hábitos de consumo:

Para analizar el efecto de las 9 variables de riesgo (Tabla 2) sobre los hábitos de consumo (edad de inicio, intención de consumo en el próximo año y frecuencia de consumo en la última semana), se llevó a cabo un análisis de regresión lineal múltiple para cada una de las sustancias estudiadas y se incluyó el sexo de los jóvenes con el fin de mantener controlada esta variable. Las tablas 4 y 5 muestran los coeficientes beta estandarizados de cada una de las variables de riesgo, cuando se toman los hábitos de consumo como variable dependiente, para cada grupo de edad (15 y a 16 años y 17 a 18 años). Teniendo en cuenta la alta correlación existente entre la escala de "riesgo personal" y la de "riesgo a terceros", solo se tuvo en cuenta para los análisis la escala de riesgo personal, con el fin de evitar efectos de colinealidad.

Intenciones de Consumo (tabla 4): En general, un aumento en la percepción de placer o beneficios predice un aumento en la intención de consumo para los jóvenes de ambas ciudades y en los dos grupos de edad, a excepción de la intención de probar drogas fuertes en los adolescentes de Barcelona de 15 a 16 años y de consumir marihuana en los jóvenes de Bogotá de 17 a 18 años. La variable "facilidad para hacer estas actividades" predice un aumento en la intención de consumir alcohol y tabaco en los jóvenes de Bogotá entre los 15 y los 16 años, mientras que para los jóvenes de Barcelona predice un aumento en la intención de consumir marihuana y drogas más fuertes (cocaína, la heroína o pastillas), en los adolescentes de 17 a 18 años. Las variables de género y admiración tienen efectos respectivamente en la intención de consumo de tabaco y de alcohol para los jóvenes de Barcelona menores de 15 a 16 años. Para los jóvenes mayores de Barcelona el percibir más riesgo personal al probar drogas fuertes aumenta la intención de consumirlas. Para los jóvenes mayores de Bogotá percibir que las personas de su edad no sienten miedo de probar drogas fuertes, aumenta su intención de probarlas.

Tabla 4. Coeficientes beta estandarizados de regresión para Intención de consumo para los dos grupos de edad (15 a 16 años

\section{5 a 16 años}

\begin{tabular}{|c|c|c|c|c|c|c|c|c|}
\hline \multirow{2}{*}{ Escala } & \multicolumn{2}{|c|}{ Bebidas alcohólicas } & \multicolumn{2}{|c|}{ Cigarrillo } & \multicolumn{2}{|c|}{ Marihuana } & \multicolumn{2}{|c|}{ Fuertes } \\
\hline & Barcelona & Bogotal & Barcelona & Bogotal & Barcelona & Bogota & Barcelona & Bogotá \\
\hline Género & 0,07 & 0,13 & $0,22^{* *}$ & 0,03 & 0,11 & 0,02 & $=0,00$ & 0.02 \\
\hline Conocimiento & $-0,05$ & 0,03 & $-0,07$ & 0,00 & 0,02 & 0,02 & $-0,06$ & $-0,06$ \\
\hline Miedo & 0,10 & $-0,09$ & $-0,05$ & 0,00 & 0,01 & 0,01 & $-0,18$ & $-0,00$ \\
\hline Riesgo personal & $-0,01$ & $-0,06$ & $-0,01$ & $-0,12$ & $-0,04$ & $-0,09$ & $-0,19$ & $-0,04$ \\
\hline Placer o Beneficios & $0,38^{* \cdots *}$ & $0,21^{\ldots \ldots}$ & $0,49^{* \cdots}$ & $0,36^{\cdots \cdots}$ & $0,41^{\ldots \ldots *}$ & $0,32^{* . *}$ & 0.22 & $0.29 * \cdots$ \\
\hline Presión & 0,02 & 0,05 & $-0,07$ & 0,02 & 0,07 & $-0,03$ & $=0,03$ & 0,00 \\
\hline Admiración & $-0,26^{* *}$ & $-0,12$ & $=0,07$ & $-0,08$ & $-0,15$ & $-0,01$ & 0,02 & $-0,01$ \\
\hline Evitabilidad & 0,01 & $-0,07$ & $-0,05$ & 0,04 & 0,20 & 0,03 & $-0,16$ & $-0,01$ \\
\hline Facilidad para hacerlo & 0,16 & $0,32^{* \ldots *}$ & 0,09 & $0,23^{* \cdots}$ & 0,15 & 0.23 & 0,16 & -0.03 \\
\hline R - Cuadrado Corregaida & 0.24 & 0.23 & 0.29 & 0.25 & 0.29 & 0.24 & 0.12 & 0.06 \\
\hline \multicolumn{9}{|l|}{17 a 18 aก̃os } \\
\hline Género & 0,02 & $-0,11$ & 0,09 & 0,03 & 0,08 & 0,06 & 0,12 & 0.09 \\
\hline Conocimiento & 0,07 & 0,15 & 0,01 & 0,06 & $-0,05$ & $-0,04$ & 0,03 & 0,19 \\
\hline Miedo & $-0,08$ & $-0,00$ & 0,01 & 0,02 & -0.04 & $-0,04$ & 0,01 & $-0,34^{* *}$ \\
\hline Riesgo personal & $-0,14$ & $-0,09$ & 0,03 & $-0,15$ & $-0,14$ & $-0,18$ & $-0,16^{* *}$ & 0.04 \\
\hline Placer o Beneficios & $0,35^{* \ldots *}$ & 0,18 & $0,41^{\cdots \cdots}$ & $0,33^{* *}$ & $0,47^{\ldots \ldots *}$ & 0,24 & $0,36^{* * *}$ & $0.29 * *$ \\
\hline Presión & 0,02 & 0,01 & $-0,02$ & 0,12 & 0,05 & 0,01 & 0,01 & $-0,06$ \\
\hline Admiracion & 0,04 & $-0,90$ & $-0,11$ & $-0,12$ & -0.03 & $-0,12$ & 0,02 & $-0,17$ \\
\hline Evitabilidad & 0,01 & $-0,03$ & $-0,10$ & 0,07 & $-0,08$ & $-0,07$ & $-0,03$ & 0,06 \\
\hline Facilidad para hacerlo & 0.01 & 0,18 & 0,11 & 0,13 & $0.18^{* *}$ & 0,14 & $0.21^{* *}$ & 0.07 \\
\hline R - Cuadrado Corregida & 0.15 & 0.08 & 0.21 & 0,19 & 0.31 & 0.10 & 0.25 & 0.17 \\
\hline
\end{tabular}

${ }^{* *} p<0.001,{ }^{* *} p<0.01$ 
Frecuencia de Consumo última semana (tabla 5): Nuevamente la escala de Placer o Beneficios tiene un efecto en la frecuencia con que se consumieron algunas de las sustancias la semana anterior a la encuesta. Es de resaltar que en los jóvenes entre los 15 y 16 años de ambas ciudades, el percibir placer o beneficios en el consumo de drogas fuertes (heroína, cocaína o éxtasis), no predice la frecuencia de su consumo. Ocurre lo mismo para el consumo de marihuana en los jóvenes de Bogotá del grupo de edad anteriormente mencionado. Sin embargo para el grupo de jóvenes entre 17 y 18 años, el percibir placer o beneficios solo predice la frecuencia de consumo de todas las sustancias en los adolescentes de Bar- celona, donde se evidencia una relación directa entre la mayor percepción de placer o beneficios y la frecuencia del consumo. Hay que resaltar que la variable "facilidad para hacerlo" tiene un claro efecto positivo en la frecuencia en que se consumió tabaco en ambas ciudades para los jóvenes mayores y entre los jóvenes menores el efecto solo fue significativo para los jóvenes de Bogotá. Como puede observarse en la tabla 5, el ser hombre tiene un efecto positivo en la frecuencia de consumo de bebidas alcohólicas en los jóvenes mayores de Barcelona. Para los de Bogotá el percibir que se admira a las personas consumidoras de alcohol de su edad, tiene un efecto negativo en el consumo del mismo.

Tabla 5. Coeficientes beta estandarizados de regresión para Frecuencia de uso en la última semana para los dos grupos de edad (15 a 16 años y 17 a 18 años)

\section{5 a 16 años}

\begin{tabular}{|c|c|c|c|c|c|c|c|c|}
\hline \multirow{2}{*}{ Escala } & \multicolumn{2}{|c|}{ Bebidas alcohólicas } & \multicolumn{2}{|c|}{ Cigarrillo } & \multicolumn{2}{|c|}{ Marihuana } & \multicolumn{2}{|c|}{ Fuertes } \\
\hline & Barcelona & Bogota & Barcelona & Bogoth & Barcelona & Bogota & Barcelona & Bogota \\
\hline Género & $-0,03$ & $-0,05$ & 0.15 & $-0,04$ & 0,10 & $-0,00$ & $-0,03$ & 0,11 \\
\hline Conocimiento & $-0,03$ & $-0,07$ & 0,03 & 0,04 & 0,12 & 0.02 & 0,16 & $-0,07$ \\
\hline Miedo & $-0,01$ & 0,09 & $-0,09$ & $-0,08$ & -0.21 & -0.07 & -0.24 & $-0,04$ \\
\hline Riespo personal & $-0,07$ & $-0,09$ & 0,08 & $-0,07$ & 0,09 & 0,06 & 0,08 & 0,01 \\
\hline Placer o Beneficios & $0,39^{* \ldots *}$ & $0,21^{* *}$ & $0,50^{* * *}$ & $0,32^{* \ldots *}$ & $0,37^{* * *}$ & 0,31 & 0,12 & 0,02 \\
\hline Presibon & $-0,09$ & 0,09 & 0.01 & 0,02 & 0,10 & -0.08 & -0.06 & 0,01 \\
\hline Admiracibn & $-0,01$ & $-0,09$ & 0,16 & $-0,15$ & 0,00 & $-0,08$ & $-0,14$ & $-0,02$ \\
\hline Evitabilidad & 0.06 & 0,07 & 0.01 & 0,01 & 0.20 & 0,00 & 0,10 & $=0,13$ \\
\hline Facilidad para hacerlo & 0,12 & 0,11 & 0.14 & $0,21^{\cdots \cdots}$ & $=0.03$ & 0.10 & 0.14 & 0,15 \\
\hline R - Cuadrado Corregida & 0.14 & 0.09 & 0.29 & 0.21 & 0.20 & 0.09 & 0.07 & 0,02 \\
\hline \multicolumn{9}{|l|}{17 a 18 años } \\
\hline Género & $-0,25^{\cdots \cdots}$ & $-0,04$ & 0.03 & $-0,00$ & $-0,04$ & 0.04 & 0,02 & 0,12 \\
\hline Conocimiento & -0.03 & -0.10 & 0.05 & 0,08 & 0,02 & 0,12 & $=0,03$ & 0,03 \\
\hline Miedo & $-0,03$ & 0,14 & 0.02 & $-0,04$ & $-0,03$ & -0.24 & 0,05 & $-0,11$ \\
\hline Riesqo personal & $-0,04$ & $-0,01$ & 0,05 & $-0,24$ & $-0,11$ & $-0,04$ & $-0,12$ & $-0,16$ \\
\hline Placer o Beneficios & $0.22^{* *}$ & 0,15 & $0.40^{* 1 *}$ & 0,18 & $0,34^{* * *}$ & 0.07 & $0,32^{* * *}$ & $-0,14$ \\
\hline Presion & -0.08 & 0,17 & -0.09 & $-0,03$ & 0.08 & 0,12 & 0,06 & $-0,06$ \\
\hline Admiracion & 0.04 & $-0,32^{* *}$ & $=0,09$ & $-0,09$ & $-0,05$ & 0,04 & 0,02 & $-0,08$ \\
\hline Evitabilidad & 0,02 & 0,14 & $-0,11$ & $-0,05$ & 0,01 & -0.21 & 0,06 & 0,10 \\
\hline Facilidad para hacerlo & 0.01 & 0,18 & $0.19^{* *}$ & $0.27^{* *}$ & 0.16 & -0.03 & 0.10 & -0.03 \\
\hline R - Cuadrado Corregida & 0.10 & 0.11 & 0.24 & 0.18 & 0.22 & 0.01 & 0.16 & 0,00 \\
\hline
\end{tabular}

${ }^{* *} \mathrm{p}<0.001,{ }^{* *} \mathrm{p}<0.01$

\section{DISCUSIÓN}

El presente estudio intentó identificar los diferentes hábitos de consumo de sustancias legales e ilegales que tienen los jóvenes de las ciudades de Barcelona (España) y Bogotá (Colombia) con el fin de determinar si los patrones de consumo de diferentes sustancias son equivalentes para los adolescentes en general o si existen diferencias según el lugar en el que se vive. Adicionalmente, se intentó analizar la influencia de las variables de riesgo percibido sobre los hábitos de consumo en los jóvenes, según la ciudad de residencia.
Los resultados obtenidos sugieren que existen diferencias en cuanto a la edad en que se inicia el consumo de alcohol, en la edad en que se embriagan los jóvenes por primera vez y la edad a la que se fuman el primer cigarrillo de marihuana, entre los jóvenes de Bogotá y de Barcelona. Sin embargo esta diferencia en la edad en que se inicia el consumo de marihuana solo se presenta para los jóvenes de 17 a 18 años. Este fenómeno sugiere que en Bogotá se está presentando un descenso en la edad de inicio de consumo de marihuana asimilándose a la edad de inicio de los jóvenes de Barcelona, pues los jóvenes mayo- 
res de Bogotá afirman haberse fumado su primer cigarrillo de marihuana cerca de los 15 años, mientras que los menores dicen haberlo hecho cerca de los 14. Adicionalmente, parece que existe un inicio similar en el consumo de tabaco y de drogas como la cocaína, la heroína o las pastillas en ambas ciudades. Los resultados mostraron que los jóvenes de Bogotá son quienes comienzan a beber alcohol y se embriagan a una edad significativamente menor que los de Barcelona. Esto, según autores como Kulbok y Cox (2002) y Wills, Walker y Resko (2005), debería suponer un mayor consumo por parte de los jóvenes de Bogotá en cuanto a las otras sustancias (marihuana, cocaína, heroína). Sin embargo esto no se confirma en este estudio, pues son los jóvenes de Barcelona los que consumen en una frecuencia mayor que los de Bogotá todas las sustancias, incluyendo el alcohol. Es posible que esto ocurra debido a que los jóvenes de Barcelona perciben mayores beneficios o placer al consumir o probar las diferentes sustancias, mientras que los de Bogotá perciben mayores posibilidades de enfermarse. Igualmente en futuras investigaciones habría que tenerse en cuenta también la percepción que tienen los jóvenes en cuanto al consumo de sus pares, ya que como proponen D'Amico y McCarthy (2006), el percibir que los pares consumen alcohol se asocia a un inicio en el alcohol.

En cuanto a las diferencias en la forma en que los jóvenes de ambas ciudades perciben las actividades de riesgo como el beber alcohol, fumar marihuana o tabaco y probar drogas fuertes, se ha constatado que hay una intención y un consumo real más alto en Barcelona que en Bogotá en cuanto a la mayoría de las sustancias, al menos en adolescentes escolarizados. Como lo evidencian los datos encontrados en este estudio, la variable de percepción de placer o de beneficios es la que explica, en mayor grado, la intención de consumo de las cuatro sustancias estudiadas. Además, la intención de consumo de alcohol y de tabaco, en los adolescentes de Bogota, se explica por la percepción de placer y simultáneamente por la percepción de facilidad de consumo; la intención de consumo de drogas fuertes se explica también por la percepción de placer y además, en forma de protección, por el miedo a los riesgos asociados al consumo de estas drogas fuertes. En los adolescentes de Barcelona, el mayor consumo de marihuana y de drogas fuertes se explica por la percepción del placer, por la facilidad de acceso a este consumo, y además, en el caso específico de las drogas fuertes, por la ausencia de percepción de riesgo personal asociado a este tipo de consumo. Esto confirma lo encontrado por otros autores como Benthin et al. (1993) y Calafat et al. (2001), los cuales sugieren que las percepciones van a determinar el comportamiento de riesgo.
Es importante resaltar que aunque la literatura sugiere que la intención puede predecir el consumo actual, en este estudio encontramos que la intención de beber alcohol en un futuro cercano por parte de los jóvenes mayores de Bogotá es un poco mayor que la de los jóvenes de Barcelona pero el consumo real es menor por parte de los jóvenes entre los 17 y los 18 años de Bogotá. Para todo el resto de sustancias estudiadas la intención de consumirlas es mayor por parte de los jóvenes de Barcelona y el consumo real también es mayor. Valdría la pena profundizar en las razones culturales que llevan a que se den estas diferencias en los hábitos de consumo de los jóvenes de ambas ciudades. Posiblemente, como afirma González, García-Señorán y González (1996), los modelos sociales son elementos que juegan un papel importante en la iniciación del consumo de drogas. Según estos autores, ciertos hábitos y estilos de vida tienden a verse como conductas de prestigio social o expresiones de placer, lo cual es altamente deseado por los adolescentes que tienden a imitarlas.

En este estudio se quiso determinar para cada sustancia psicoactiva estudiada qué variables de riesgo podrían predecir la intención y la frecuencia de consumo. Los datos obtenidos sugieren que el placer o los beneficios percibidos influyen de modo consistente y evidente en la intención de consumir y en la frecuencia de su uso, tanto para Barcelona, como para Bogotá en la mayoría de las sustancias. Esto confirma lo encontrado por Parker et al., (1998), quienes sugieren que las decisiones sobre usar o no sustancias psicoactivas están relacionadas con la percepción de beneficios y riesgos. Sin embargo hace falta profundizar en las razones por las que el placer o beneficio no predice la frecuencia de consumo de ninguna de las sustancias en los jóvenes mayores de Bogotá.

Parsons, Siegel y Cousins (1997) indican que en los adolescentes mayores, los beneficios percibidos son mejores determinantes de la intención de consumir una sustancia que los riesgos percibidos. En este estudio se confirma lo propuesto por los autores ya que los resultados sugieren que mientras los beneficios percibidos si predicen de forma significativa la intención o la frecuencia de consumo de la mayoría de las sustancias, el percibir posibles riesgos asociados al uso de estas sustancias no tuvo efectos significativos para ninguno de los grupos estudiados, a excepción de la intención de consumir drogas fuertes en Barcelona para el grupo de jóvenes entre 17 y 18 años. Para este grupo percibir que ellos mismos o sus compañeros estén en mayor riesgo de enfermarse o herirse también predice una mayor intención de consumo. 
Por otro lado, la literatura sugiere que en cuanto a la frecuencia de consumo, la facilidad (entendida como la accesibilidad a la sustancia, la presencia de esta y la aceptación de su uso) se asocia a la prevalencia, cantidad y frecuencia de consumo argumentando que cuanta más facilidad se percibe, mayor es el consumo (Makkai y McAllister, 1998; Johnston, etl al., 2004). En este estudio, la frecuencia de consumo únicamente es predecible a partir de la percepción de facilidad para el tabaco en ambas ciudades para los jóvenes mayores y en los jóvenes menores sólo para Bogotá. Esto sugiere que la relación explicativa entre facilidad y frecuencia de consumo es sólo cierta para el tabaco, cuya disponibilidad es ciertamente elevada.

La escasa relevancia que la presión de los iguales alcanza en la intención y frecuencia de consumo lleva a pensar que los adolescentes, en su tendencia a aceptar los comportamientos grupales como medio de identificación, no son conscientes de la presión que el propio grupo ejerce sobre ellos.

Hay que tener en cuenta que una limitación de este estudio es el diseño, pues todas las mediciones se tomaron a partir de cuestionarios de auto reporte. En futuras investigaciones podría incluirse una medición objetiva del fenómeno, tal como la obtención de información por parte de los amigos, los padres o los profesores y así poder comparar las respuestas de los jóvenes con la que dan los otros informantes.

Los resultados del presente estudio tienen implicaciones para el desarrollo de programas de prevención centrados en adolescentes escolarizados. Como pudo observarse, el placer o los beneficios percibidos parecen influir en la intención de uso y en la frecuencia de consumo de las sustancias tanto legales como ilegales en ambas ciudades. Es entonces necesario que las actividades dirigidas a prevenir el uso de sustancias, centren parte de sus esfuerzos en desmitificar los supuestos resultados beneficiosos de consumir sustancias. Por otro lado, variables como la percepción que se tiene sobre la facilidad de llevar a cabo actividades como consumir alcohol los fines de semana, o fumar con regularidad tabaco o marihuana y probar drogas como la cocaína, la heroína o las pastillas no necesariamente predicen la intención ni la frecuencia de consumo y esto dependerá del grupo de edad con el que se trabaje y de las ciudad donde viven los jóvenes y el tipo de sustancia de la que se trate. Así, según lo encontrado en este estudio, los programas de prevención tendrán que tener en cuenta el tipo de sustancia con el que se esté trabajando, la edad del joven y la ciudad en la que vive, para tratar variables como la percepción del miedo a las consecuencias de usar drogas, el riesgo de hacerse daño, la percepción que tienen los jóvenes sobre la evitación de sus com- pañeros de situaciones de riesgo, pues todas estas variables tienen efectos diferentes sobre las intenciones y el uso real de las diferentes sustancias para cada grupo.

\section{AGRADECIMIENTOS}

Esta investigación la ha apoyado el Departament d'Universitats, Recerca i Societat de la Informació de la Generalitat de Catalunya y del Fondo Social Europeo, mediante una beca otorgada al primer autor.

También ha sido financiada por el MCyT mediante la beca SEJ 2005 - 09144 - C02 - 01.

\section{REFERENCIAS}

Amengual, M., Calafat, A., y Palmer, A. (1993). Alcohol, tabaco y drogas en enseñanza media. 1981-1988-1992. Adicciones, Vol.5, 2, 141-161.

Ballester, R., Gil, M.D., Girardo, M.C. (2000). Comportamientos y Actividades Relacionadas con el Consumo de Alcohol en Adolescentes de 15 a 17 años. Análisis y Modificación de la Conducta. 26(110), 835 - 851.

Benthin, A., Slovic, P., y Severson, H. (1993). A psychometric study of adolescent risk perception. Journal of adolescence, 16, $153-168$.

Calafat, A., Cesáreo, F., Juan, M., Bellis, M.a., Bohrn, K., Hakkarainen, P., Kilfoyle-Carrington, M., Kokkevi, A., Maalsté, N., Mendes, F., Siamou, I., Simon, J., Stocco, P. y Zavatti, P. (2001). Risk and Control in the Recreational Drug Culture. Sonar 01. Palma de Mallorca: IREFREA.

Calafat, A., Stocco, P., Mendes, F., Simon, J., Van de Wijngaart, G., Sureda,P. et al. (1998) Characteristics and Social Representation of Ecstasy in Europe. Palma de Mallorca: IREFREA and European Commision.

D'Amico, E.J., y McCarthy, D.M. (2006). Escalation and Initiation of Younger Adolescents' Substance Use: The Impact of Perceived Peer Use. Journal of Adolescent Health. $39481-487$.

Fountain, J., Griffiths, P., Farrell, M., Gossop, M., y Strang, J. (1999). Benzodiazepines in polydrug-using repertoires: the impact of the decreased availability of temazepam gel-filled capsules. Drugs: Education, Prevention and Policy, 6, 61 - 69.

González, F., García-Señorán y González S. (1996). Consumo de drogas en la adolescencia. Psicothema 8, 257 $-267$.

Hampson, S. E., Severson, H. H., Burns, W. J., Slovic, P., y Fisher, K. J. (2001). Risk perception, personality factors and alcohol use among adolescents. Personality and Individual Differences, 30, 167 - 181.

Jessor, R., Van Den Bos, J., Banderín, J., Costa, F. y Turbin, S. (1995). Protective Factors in Adolescent Problem 
Behavior: Moderator Effects and Developmental Change. Developmental Psychology 31, 923 - 933.

Johnston, L.D., O'Malley, P.M., Bachean, J.G. y Schulenberg, J.E. (2004). Monitoring the Future National Results on Adolescent Drug Use: Overview of Key Findings, 2003. (NIH Publication No. 04-5506). Bethesda, MD: National Institute on Drug Abuse.

Kulbok, P.A., y Cox, C.L. (2002). Dimensions of adolescent health behavior. Journal of Adolescente Health, 31 393-400.

Makkai, T., y McAllister, I. (1998). Patterns of Drug Use in Australia 1985 - 95 (No. 2205). Canberra: Commonwealth Department of Health and Family Services.

Mayock, P. (2002). Drug Pathways, transitions and decisions: The experiences of Young People in an inner-city Dublin Community. Contemporary Drug Problems, 29, 117 - 141.

Morgan, M. y Grube, J. (1991) Closeness and peer group influence. Brithish Journal of Social Psycholgy 30, 159 $-169$

Parker, H., Aldridge, J., y Measham, F. (1998). Illegal Leisure: the normalisation of adolescent recreational drug use. London: Routledge.
Parsons, J. T., Siegel, A. W., y Cousins, J. H. (1997). Late adolescent risk taking: effects of Perceived Benefits and Perceived risks on behavioral interactions and behavioral change. Journal of adolescence, 20, 381 - 392.

Plan Nacional Sobre Drogas, PNSD, ed (2004). Informe 2004. Observatorio Español Sobre Drogas. Ministerio de Sanidad y Consumo, Madrid.

Rumbos (2001). Encuesta Nacional sobre Consumo de Sustancias Psicoactivas en jóvenes de 10 a 24 años. Observatorio Colombiano sobre consumo de Sustancias Psicoactivas.

Slovic, P. (1987). Perception of Risk. Science, 236, 280 - 285.

Severson, H., Slovic, P., Hampson, S., y Schrader, L. (1990). Adolescent risk perception: A measure to further our understanding of tobacco and drug use. Hygie, 9, 27-29.

UNODC (2006). World Drug Report 2006. Viena: Naciones Unidas.

Wills, T.A., Walker, C., y Resko, J.A. (2005). Longitudinal Studies of Drug Use and Abuse. En Z. Sloboda (Ed). Epidemilogy of Drug Abuse. Springer, New York.. 
